\title{
Influence of Multi-hole Dislocation Distribution on the Characteristics of Graphite Nanoribbons
}

\author{
Wei Chun ${ }^{1}$, Li Ming ${ }^{2}$, Yao Ming' ${ }^{1}$,Wei Wanhua ${ }^{1}$, Wu Zhengnan ${ }^{1}$ \\ ${ }^{1}$ School of Electronic and Information Engineering, Wuhan Donghu University, Wuhan 430212, China \\ ${ }^{2}$ Information Management Center, Air Force Early Warning Academy, Wuhan 430019, China
}

Keywords: graphene nanoribbons; dislocation distribution; Green's function; heat transfer; multi-hole

\begin{abstract}
The graphene is a newly emerging material in recent years. The Nobel Prize in physics in 2010 was awarded to the discoverers of graphene, Geim and Novoselov, which aroused the researchers' interest in graphene, making it a most brilliant new star in the field of nano material science. In order to analyze the influence of the multi-space acupoint distribution on the properties of graphite nanometers, the mathematical model of the misdistribution of the multi space acupoint in graphite nanoscale was established by using the nonequilibrium Green function method. The effect of the three hole dislocation distribution on the thermal transport properties in the graphite nanoscale was studied. Finally, the distribution of different types of dislocation was carried out. Test. From the experimental results, the multi-space acupoint error has a certain influence on the thermal transmission characteristics of the graphite nanoscale, and the effect of rotating dislocation is more obvious than that of the shift dislocation, which provides an effective theoretical basis for the design of the thermal transport quantum devices based on the graphite nanometers.
\end{abstract}

\section{Introduction}

Since the end of the twentieth Century, various carbon based nanomaterials have attracted the attention of researchers because of their unique structure, properties and wide application prospects. Zero dimensional fullerenes (C60), one dimensional carbon nanotubes (CNTs) and two-dimensional graphene (graphene) have been developed in succession in chemistry, physics, material science and other related disciplines. Graphene is a two-dimensional material consisting of a carbon atom with a hybrid orbital and a six angle honeycomb lattice with only one carbon atom thickness. The shape of graphene is very thin, but the hardness is very high, the transparency is very good, the absorption rate of light is very low, the thermal conductivity is good, the thermal conductivity exceeds the diamond and the carbon nanotube, the graphene material has higher mobility and lower resistivity, its mobility is higher than the carbon nanotube and silicon crystal, and the resistivity is higher than copper. Materials and silver materials are lower, the material with the lowest resistivity at present. Because of the low resistivity of graphene, the conductive velocity is very fast, so the ultrathin electronic components and electrohydraulic crystal materials can be developed. It has a very good application prospect.

\section{The Structure and Properties of the Graphene Energy Band}

In recent years, with the development of micromachining technology, the low dimension nanostructures of lower than 30nm have been produced. Graphene is a new type of single atomic layer of carbon nanomaterials. The structure of the electronic energy band of graphene has a great influence on its characteristics, because its overall structure is based on the independent carbon atom, and the perturbation is produced by the surrounding carbon atoms. The energy level distribution of graphene can be analyzed by matrix calculation, and the approximate two quantized Hamiltonian can be used to represent the graphite electron tight binding model, as shown in formula (1). 


$$
H=\varepsilon_{2 p z} \sum_{i}\left(a_{i}^{+} a_{i}+b_{i}^{+} b_{i}\right)-t \sum_{<i, j>}\left(a_{i}^{+} b_{j}+h . c\right)
$$

The influence of electron spin is neglected in the formula, in which $<i, j>$ means that the sum of the electron transitions to the adjacent lattice, $\varepsilon_{2 p z}$ is the orbit energy of the single electron $2 p z$.

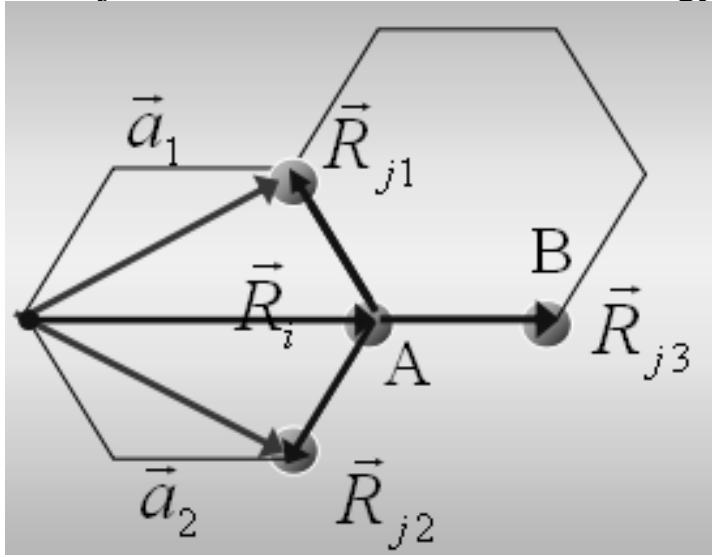

Fig.1 The adjacent atomic schematic diagram of a reference atom

As shown in Figure 1, the graphite lattice can be represented by the adjacent two carbon atoms A,B , which are not equivalent to the geometric environment of the two carbon atoms. The selected atom A is used as a reference atom, and its vector is $\vec{R}_{i}$, the vector of the atom adjacent to it is $\vec{R}_{j 1}, \vec{R}_{j 2}$ and $\vec{R}_{j 3}$.It is assumed to $a_{i}^{+}\left(b_{j}\right)$ be an laocated $\vec{R}_{i}\left(\vec{R}_{j}\right)$ electronic generation or elimination operator in the formula (1). It is known that the transition energy $b_{j}$ can be seen $\vec{R}_{j}$ from the transition $\vec{R}_{i}$ to the nearest neighbor $a_{i}^{+}$and the transition energy $t=2.8 e V$ by Fourier transformationtransformation.

$$
\begin{gathered}
a_{i}=\frac{1}{\sqrt{N}} \sum_{\vec{k}} e^{i \vec{k} \cdot \vec{R}_{i}} \hat{a}_{k} \\
b_{j}=\frac{1}{\sqrt{N}} \sum_{\vec{k}} e^{i \vec{k} \cdot \vec{R}_{j}} \hat{b}_{k} \\
\left|\begin{array}{cc}
\varepsilon_{2 p z}-E & -t\left(1+e^{-i \vec{k} \cdot \vec{a}_{1}}+e^{-i \vec{k} \cdot \vec{a}_{2}}\right) \\
-t\left(1+e^{i \vec{k} \cdot \vec{a}_{1}}+e^{i \vec{k} \cdot \vec{a}_{2}}\right) & \varepsilon_{2 p z}-E
\end{array}\right|=0
\end{gathered}
$$

In the case of an ideal structure model, graphene can be selected as the reference point of energy $E(k)$ with the atomic orbital energy level $\varepsilon_{2 p z}=0$, and the energy band $\pi$ of the graphene atom can be obtained from the formula.

$$
\begin{aligned}
& E^{2}=t^{2}\left(1+e^{i \vec{k} \cdot \vec{a}_{1}}+e^{i \vec{k} \cdot \vec{a}_{2}}\right)\left(1+e^{-i \vec{k} \cdot \vec{a}_{1}}+e^{-i \vec{k} \cdot \vec{a}_{2}}\right) \\
& =t^{2}\left\{3+2 \cos \left(\vec{k} \cdot \vec{a}_{1}\right)+2 \cos \left(\vec{k} \cdot \vec{a}_{2}\right)+2 \cos \left[\vec{k} \cdot\left(\vec{a}_{1}-\vec{a}_{2}\right)\right]\right\}
\end{aligned}
$$

In which,

$$
\vec{k} \cdot \vec{a}_{1}=\left(k_{x}, k_{y}\right)\left(\frac{3 a}{2}, \frac{\sqrt{3} a}{2}\right)=\frac{3 a}{2} k_{x}+\frac{\sqrt{3} a}{2} k_{y}
$$




$$
\begin{gathered}
\vec{k} \cdot \vec{a}_{2}=\frac{3 a}{2} k_{x}-\frac{\sqrt{3} a}{2} k_{y} \\
\vec{k} \cdot\left(\vec{a}_{1}-\vec{a}_{2}\right)=\sqrt{3} a k_{y}
\end{gathered}
$$

After elementary trigonometric function and difference operation, graphene energy band is obtained.

$$
E_{ \pm}\left(k_{x}, k_{y}\right)= \pm t \sqrt{3+2 \cos \left(\sqrt{3} k_{y} a\right)+4 \cos \left(\frac{\sqrt{3} k_{y} a}{2}\right) \cos \left(\frac{3 k_{x} a}{2}\right)}
$$

The energy band of graphene can be obtained from the calculation formula, and the structure and characteristics of graphene nanoribbon FET can be analyzed with the aid of computer aided software.

\section{The Theoretical Model of Multi-hole Graphite Nanometers}

The whole structure of the multi hole graphite nanoribbons is based on the independent carbon atom, and the perturbation is produced by the surrounding carbon atoms. Because of the existence of the cavitation, some characteristics of the nanoscale can be changed, such as the heat transfer characteristics, a three hole graphite nanoscale structure model, as shown in Figure 2.

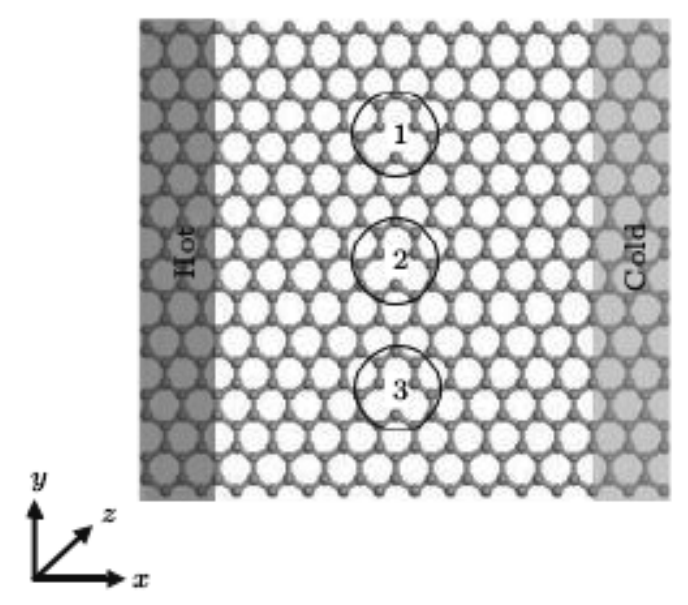

Fig.2 The structure schematic diagram of multi hole graphite nanoscale

As shown in Figure 2, there is a hole structure in the nanoscale structure. The effect of the hole structure on the thermal transport properties of nanoscale is mainly studied. The hole area in the map is divided into three. According to the Landauer formula and the linear response hypothesis, the thermal transport equation in the nanoscale can be written as the equation.

$$
J_{i}=\frac{h}{2 \pi} \int_{0}^{\infty} \tau_{i}(w) w\left(f_{L}(w)-f_{R}(w)\right) d_{w}
$$

It is the distribution function of the phonon in the graphite nanoscale $f_{L}(w)=1 /\left[\exp \left(h_{w} / k_{B} T_{L / R}\right)\right]$, indicating the transmission coefficient $\tau_{i}(w)$. In the left side, the temperature $T_{1}$ is the energy input heat sink, the middle part is the phonon scattering area, the right is the time energy output heat sink, and the average temperature $T_{2}$ can be used directly at the temperature $T$ difference to zero. Under the condition of neglecting the electro acoustic interaction, the formula of thermal conductivity can be approximately written. 


$$
k_{i}=\frac{h^{2}}{k_{B} T^{2}} \frac{1}{2 \pi} \int_{0}^{\infty} \tau_{i}(w) \frac{w^{2} e^{\beta w}}{\left(e^{\beta w}-1\right)^{2}} d w
$$

In which, $\beta=1 /\left(k_{B} T\right)$.The study shows that the graphite nanometers can be divided into two models, one of which is the IPMs model, the other is FPMs, which ignores the coupling of the model, and its Hamilton equation is the same.

$$
\text { Hsys }=\text { Hsys, } x-y+\text { Hsys, } z
$$

The thermal conductivity and molecular projection of graphite nanoribbons can be decomposed into thermal conductivity and projection probability of IPMs and FPMs, which can be calculated independently. Under the condition of harmonic approximation, the Hamiltonian equation of FPMs can be expressed as

$$
H_{s y s, z}=\sum_{\alpha=L, C, R} H_{\alpha, z}+\left(u_{z}{ }^{L}\right)^{T} K_{z}{ }^{L C} u_{z}{ }^{C}+\left(u_{z}{ }^{C}\right)^{T} K_{z}{ }^{C R} u_{z}{ }^{R}
$$

The Hamilton equation of IPMs is

$$
H_{s y s, x-y}=\sum_{\alpha=L, C, R} H_{\alpha, x-y}+\left(u_{x-y}{ }^{L}\right)^{T} K_{x-y}{ }^{L C} u_{x-y}{ }^{C}+\left(u_{x-y}{ }^{C}\right)^{T} K_{x-y}{ }^{C R} u_{x-y}{ }^{R}
$$

For FPMs, the dynamic moment of a complete linear system can be expressed as

$$
K z=\left(\begin{array}{ccc}
k_{z}{ }^{L} & k_{z}{ }^{L C} & 0 \\
k_{z}{ }^{L C} & k_{z}{ }^{C} & k_{z}{ }^{C R} \\
0 & k_{z}{ }^{R C} & k_{z}{ }^{R}
\end{array}\right)
$$

The IPMs dynamic moment can be expressed as

$$
K_{x-y}=\left(\begin{array}{ccc}
k_{x-y}{ }^{L} & k_{x-y}{ }^{L C} & 0 \\
k_{x-y}{ }^{L C} & k_{x-y}{ }^{C} & k_{x-y}{ }^{C R} \\
0 & k_{x-y}{ }^{R C} & k_{x-y}{ }^{R}
\end{array}\right)
$$

Through the unbalanced Green function method, the PFMs's Green function can be written.

$$
g_{L(R), z}^{r}=\left[(w+i \eta)^{2} I_{z}-K_{L(R), z}\right]^{-1}
$$

The Green function of IPMs is

$$
g_{L(R), x-y}^{r}=\left[(w+i \eta)^{2} I_{x-y}-K_{L(R), x-y}\right]^{-1}
$$

It is known from the above analysis that in order to calculate the thermal conductivity, it is the key to calculate the transmittance ${ }^{\tau_{i}}$ of the phonons, and the effect of the misdistribution of the multiple acupoints on the thermal transport characteristics can be calculated.

Influence of space point acupoints on characteristics of graphite nanoribbons

In order to explore the effect of multi space acupoint distribution on the characteristics of graphite nanoscale, this study was conducted to test the effect of different acupoint distribution on the thermal transport of graphene nanoribbons. The reduction of thermal conductivity under different temperature conditions was obtained, as shown in Figure 3. 


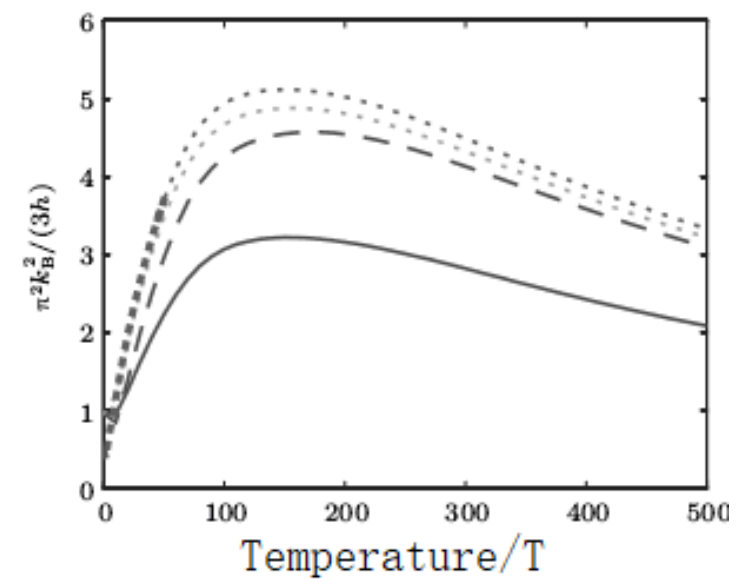

Fig. 3 The reduction of thermal conductivity of IPMs and FPMs with rotational dislocation

The solid line represents the performance curve of the three hole normal distribution, and the dot line and dot line respectively indicate the hole rotation 45 degree and 90 degree, and the 90 degree rotation downward. It is found that the rotation dislocation has a certain influence on the thermal transport performance of IPMs and FPMs. In order to further study the law of the influence, the thermal conductivity is tested and calculated at different translational dislocations, and the results as shown in Figure 4 are obtained.

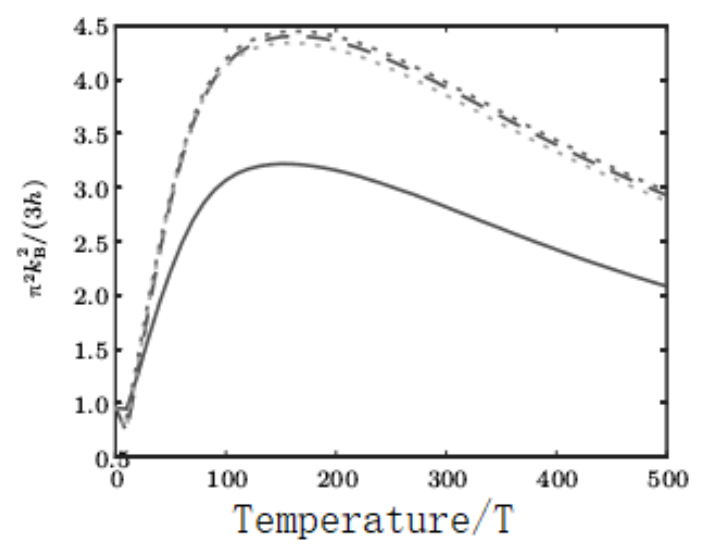

Fig.4 The reduction of thermal conductivity of translational dislocations IPMs and FPMs

The solid line represents the performance curve of the three hole normal distribution, and the dot line and dot line respectively denote the hole translation a and 2a and the translation 2A rotate 45 degrees. As shown in Figure 3, the reduced thermal conductivity of the translational dislocation IPMs and FPMs varies with the temperature. It is found that the translational dislocation has a certain effect on the thermal transport properties of IPMs and FPMs. Comparing it with figure 3, it is found that the effect of the shift dislocation on the graphite nanometers is greater than that of the rotating dislocation.

\section{Conclusion}

In order to study the effect of dislocation on the characteristics of graphite nanometers, the dislocation cavitation of graphite nanoribbons was analyzed in theory, and a multi space acupoint distribution model of graphite nanometers was established. Finally, the thermal transmission characteristics of the graphite nanometers by rotating dislocation and transposition error were studied by experimental test. Ringing. It is found from the experimental results that the rotating dislocation and dislocation have a certain influence on the thermal transport performance of IPMs and FPMs. Comparing the thermal conductivity of two dislocations, it is found that the effect of the shift dislocation on the graphite nanoscale is greater than that of the rotating dislocation. The 
experimental results provide technical support for the manufacture of graphite nanometers.

\section{Acknowledgement}

The work was supported by the Youth Foundation Project of Wuhan Donghu University with the project number 2017dhzk002 and the project name Principle design of graphene superlattice superlattice field effect device.

\section{References}

[1] Geim A K. Graphene: Status and Prospects[J]. Science, 2009, 324(5934):1530-1534.

[2] Shen H, Shi Y, Wang X. Synthesis, charge transport and device applications of graphene nanoribbons[J]. Synthetic Metals, 2015, 210:109-122.

[3] Wang L, Meric I, Huang P Y, Gao Q, Gao, Y, Tran H, Taniguchi T, Watanabe K, Campos L M, Muller D A. One-dimensional electrical contact to a two-dimensional material[J]. Science, 2013, 342(6158):614-617.

[4] Balog R, Jørgensen B, Nilsson L, Andersen M, Rienks E, Bianchi M, Fanetti M, Laegsgaard E, Baraldi A, Lizzit S, Sljivancanin Z, Besenbacher F,Hammer B, Pedersen T G, Hofmann P, Hornekaer L. Bandgap opening in graphene induced by patterned hydrogen adsorption[J]. Nature Materials, 2010, 9(4):315-319.

[5] Wei D, Liu Y, Wang Y, Zhang H, Huang L, Yu G. Synthesis of N-doped graphene by chemical vapor deposition and its electrical properties[J]. Nano Letters, 2009, 9(5):1752-1758.

[6] Hu Y Y, Li W Q, Yang L, Feng JK, Tian WQ. Electronic properties and nonlinear optical responses of boron/nitrogen-doped zigzag graphene nanoribbons[J]. Canadian Journal of Chemistry, 2016, 94(7):620-625.

[7] Zhuang X D, Chen Y, Liu G, Li P P, Zhu C X, Kang E T, Neoh K G, Zhang B, Zhu J H, Li Y X. Conjugated-Polymer-Functionalized Graphene Oxide: Synthesis and Nonvolatile Rewritable Memory Effect[J]. Advanced Materials, 2010, 22(15):1731-1735.

[8] Feng M, Zhan H, Chen Y. Nonlinear optical and optical limiting properties of graphene families[J]. Applied Physics Letters, 2010, 96(3):390.

[9] Feng M, Sun R, Zhan H, Chen Y. Lossless synthesis of graphene nanosheets decorated with tiny cadmium sulfide quantum dots with excellent nonlinear optical properties[J]. Nanotechnology, 2010, 21(7):75601.

[10] Wang X, Ouyang Y, Jiao L, Wang H, Xie L, Wu J, Guo J, Dai H. Graphene nanoribbons with smooth edges behave as quantum wires[J]. Nature Nanotechnology, 2011, 6(9):563-567.

[11] Dutta S, Pati S K. Edge reconstructions induce magnetic and metallic behavior in zigzag graphene nanoribbons[J]. Carbon, 2010, 48(15):4409-4413.

[12] Li X, Wang X, Zhang L, Lee S, Dai H. Chemically Derived, Ul trasmooth Graphene Nanoribbon Semiconductors[J]. Science, 2008, 319(5867):1229-1232.

[13] Schwierz F. Graphene transistors[J]. Nature nanotechnology, 2010, 5(7):487-496. 